\title{
Estudo da correlação entre os níveis de hemoglobina fetal e o prognóstico dos pacientes com anemia falciforme
}

\author{
Study of the correlation between fetal hemoglobin levels and the prognosis of patients with \\ sickle cell anemia
}

Lilianne B. Silva ${ }^{I}$

Romélia P. Gonçalves ${ }^{2}$

Michele F. Martins ${ }^{1}$

\begin{abstract}
Os niveis de hemoglobina fetal ( $H b F)$ foram analisados em 47 pacientes com anemia falciforme, residentes em Fortaleza, capital do Ceará, com a finalidade de fornecer informações sobre a influência da HbF no prognóstico desta doença. A determinação dos valores hematológicos foi realizada em contador automático de células sanguíneas (modelo Sysmex $K X-21 N$, Roche) e os níveis de HbF foram determinados pela técnica da desnaturação alcalina. Dentre os resultados foi demonstrada uma tendência de menor número de pacientes com crises vaso-oclusivas $(p=0,0405)$ e úlceras de perna $(p=0,0076)$ com o aumento dos níveis de HbF. Não houve diferença estatisticamente significativa na comparação entre os níveis de HbF e os valores de hemácias, hemoglobina, hematócrito, leucócitos e plaquetas. Os resultados demonstram que os níveis de hemoglobina fetal podem ser utilizados como fator prognóstico nos pacientes com anemia falciforme, no entanto não deve ser a única ferramenta diagnóstica para prever o quadro clínico destes pacientes. Rev. Bras. Hematol. Hemoter.
\end{abstract}

Palavras-chave: Anemia falciforme; hemoglobina fetal; prognóstico.

\section{Introdução}

A anemia falciforme é o resultado de uma mutação pontual (GAG $\rightarrow$ GTG) no códon do gene da globina $\beta$, conduzindo a uma substituição de ácido glutâmico por valina na sexta posição da cadeia polipeptídica. ${ }^{1}$ É caracterizada pela homozigose da hemoglobina S (SS). ${ }^{2}$

Os pacientes com anemia falciforme possuem um quadro clínico heterogêneo. ${ }^{3,4}$ A heterogeneidade clínica da doença está relacionada a fatores genéticos e adquiridos. Entre os fatores adquiridos destaca-se o nível socioeconômico, com as consequentes variações na qualidade de alimentação, prevenção de infecções e assistência médica. ${ }^{5,6}$ Em relação às características geneticamente determinadas, têm importância na gravidade clínica: as variações nos níveis de $\mathrm{HbF}$, a coexistência de alfa-talassemia e o tipo de haplótipo associado ao gene da $\mathrm{HbS}^{5,7,8}$

A HbF é produzida pelos genes da globina $\gamma$ e está restrita a um pequeno contingente de eritrócitos, as células F, cujo número é determinado geneticamente. Nos pacientes com anemia falciforme, as células F contêm cerca de $20 \%$ de $\mathrm{HbF}$ e $80 \%$ de $\mathrm{HbS}$, enquanto os outros eritrócitos contêm somente $\mathrm{HbS} .{ }^{9}$

O aumento dos níveis de $\mathrm{HbF}$ está associado à redução da morbidade e mortalidade da doença. ${ }^{10}$ Atualmente, diversos agentes citotóxicos (hidroxiureia e 5-azacitidina), fatores de crescimento hematopoético (eritropoetina) e ácidos graxos de cadeia curta (butirato e derivados) têm sido utilizados e amplamente estudados no tratamento da anemia falciforme, pois estimulam a síntese de $\mathrm{HbF}^{7,11}$

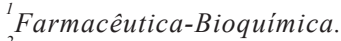

Universidade Federal do Ceará - Fortaleza-CE.

Correspondência: Lilianne Brito da Silva

Rua Dr. José Lourenço, 1820, apto. 502 - Aldeota

60115-281 - Fortaleza, CE - Brasil

E-mail: liliannebrito@hotmail.com

Doi:
}

${ }^{2}$ Farmacêtica-Bioquímica. Profa. Associada da Universidade Federal do Ceará. 
O presente estudo teve como objetivo analisar se os níveis de hemoglobina fetal podem ser utilizados como fator prognóstico nos pacientes com anemia falciforme.

\section{Casuística e Método}

Trata-se de um estudo corte transversal em 47 pacientes com diagnóstico clínico e laboratorial de anemia falciforme (forma homozigótica SS), atendidos no ambulatório do serviço de Hematologia de um hospital de referência em Fortaleza, capital do Ceará, todos voluntários, de ambos os sexos. Foram excluídos do estudo os pacientes que receberam transfusão sanguínea nos últimos três meses antes da coleta e os que faziam uso de hidroxiureia ou qualquer medicação que estimule a síntese de hemoglobina fetal. O consentimento informado foi obtido de todos os indivíduos e o estudo foi submetido e aprovado pelo Comitê de Ética da Universidade Federal do Ceará.

A determinação dos valores hematológicos foi realizada em contador automático de células sanguíneas (modelo Sysmex KX-21N, Roche). A determinação da concentração de hemoglobina fetal foi realizada pelo método de desnaturação alcalina. ${ }^{12}$

Os pacientes foram divididos em 3 grupos, de acordo com os níveis de $\mathrm{HbF}: \leq 5 \%(\mathrm{n}=16-34,04 \%),>5$ e $\leq 10$ $(\mathrm{n}=24-51,06 \%)$ e $>10 \%(\mathrm{n}=7-14,90 \%)$.

O GraphPad Prism (versão 5.0) foi o programa utilizado para a realização da análise estatística. O teste de tendência com qui-quadrado foi utilizado para correlacionar os níveis de $\mathrm{HbF}$ e as complicações clínicas dos pacientes com anemia falciforme. O teste Anova foi utilizado para a comparação entre os níveis de $\mathrm{HbF}$ e os valores de hemácia, hemoglobina, hematócrito, leucócitos e plaquetas. O nível de significância estatística considerado na análise foi $\mathrm{p}<0.05$.

\section{Resultados}

Houve uma tendência de menor número de pacientes com crises vaso-oclusivas $(\mathrm{p}=0,0405)$ e úlceras de perna $(\mathrm{p}=0,0076)$ com o aumento dos níveis de hemoglobina fetal (Tabela 1, Gráficos 1 e 2). Não houve tendência estatisticamente significativa em relação ao número de indivíduos que apresentaram os demais eventos clínicos com os níveis de hemoglobina fetal (Tabela 1).

Não houve diferença estatisticamente significativa na comparação entre os três diferentes níveis de $\mathrm{HbF}$ e os valores de $\mathrm{He}, \mathrm{Hb}, \mathrm{Ht}$, leucócitos e plaquetas (Tabela 2).

\section{Discussão}

Estudos anteriores demonstraram uma associação entre a heterogeneidade clínica da anemia falciforme e os níveis de $\mathrm{HbF}$. Um estudo que demonstrou os riscos e benefícios do uso de hidroxiureia, agente terapêutico indutor da síntese de $\mathrm{HbF}$, concluiu que a administração da droga está associada a uma redução de morbidade e mortalidade dos pacientes com anemia falciforme. ${ }^{10}$

No presente estudo foi verificada uma tendência de menor número de pacientes com crises vaso-oclusivas ( $\mathrm{p}=0,0405)$ e úlceras de perna $(\mathrm{p}=0,0076)$ com o aumento dos níveis de hemoglobina fetal. Resultado semelhante foi encontrado por Buchanan et al. (2004) e Adorno et al. (2008), que demonstraram uma associação entre níveis mais elevados de $\mathrm{HbF}$ e uma baixa frequência de úlceras da perna. Nossos

Tabela 1. Complicações clínicas dos pacientes com anemia falciforme de acordo com o nível de hemoglobina Fetal

\begin{tabular}{lcccc}
\hline & $\mathrm{HbF} \leq 5$ & $\mathrm{HbF}>5 \mathrm{e} \leq 10$ & $\mathrm{HbF}>10$ & Valor de $\mathrm{p}$ \\
\hline Crises vaso-oclusivas & $12 / 16(75 \%)$ & $14 / 24(58,33 \%)$ & $2 / 7(28,57 \%)$ & $\mathrm{A}<0 \mathrm{p}=0,0405$ \\
Transfusões de hemácias & $15 / 16(93,75 \%)$ & $18 / 24(75 \%)$ & $5 / 7(71,42 \%)$ & $\mathrm{p}>0,05$ \\
Pneumonia & $9 / 16(56,25 \%)$ & $10 / 24(41,67 \%)$ & $3 / 7(42,86 \%)$ & $\mathrm{p}>0,05$ \\
Infecção urinária & $8 / 16(50 \%)$ & $7 / 24(29,17 \%)$ & $3 / 7(42,86 \%)$ & $\mathrm{p}>0,05$ \\
Litíase biliar & $6 / 16(37,5 \%)$ & $9 / 24(37,5 \%)$ & $0 / 7(0 \%)$ & $\mathrm{p}>0,05$ \\
Insuficiência Cardíaca & $3 / 16(18,75 \%)$ & $7 / 24(29,17 \%)$ & $0 / 7(0 \%)$ & $\mathrm{p}>0,05$ \\
Acidente Vascular Cerebral & $2 / 16(12,5 \%)$ & $0 / 24(0 \%)$ & $0 / 7(0 \%)$ & $\mathrm{p}>0,05$ \\
Internação & $12 / 16(75 \%)$ & $13 / 24(54,17 \%)$ & $3 / 7(42,86 \%)$ & $\mathrm{p}>0,05$ \\
Úlceras de perna & $8 / 16(50 \%)$ & $5 / 24(20,83 \%)$ & $0 / 7(0 \%)$ & $\mathrm{A}<0 \mathrm{p}=0,0076$ \\
Necrose no fêmur & $1 / 16(6,25 \%)$ & $3 / 24(12,5 \%)$ & $0 / 7(0 \%)$ & $\mathrm{p}>0,05$ \\
\hline
\end{tabular}

Os resultados das manifestações clínicas foram reportados para cada nível de $\mathrm{HbF}$ em valores de n/total (\%), ou seja, no número de pacientes em que ocorreu o evento dividido pelo total de pacientes (porcentagem). $O$ teste utilizado foi o teste de tendência com qui-quadrado.

$\mathrm{A}<0$ - $\mathrm{A}$ tendência do evento é menor à medida que aumenta o nível de hemoglobina Fetal 
Tabela 2. Dados do hemograma de acordo com o nível de hemoglobina Fetal

\begin{tabular}{ccccc}
\hline & $\mathrm{HbF} \leq 5$ & $\mathrm{HbF}>5 \mathrm{e} \leq 10$ & $\mathrm{HbF}>10$ & Valor de $\mathrm{p}$ \\
\hline $\mathrm{He}\left(\mathrm{milhões} / \mathrm{mm}^{3}\right)$ & $2,83 \pm 0,76$ & $2,75 \pm 0,55$ & $2,47 \pm 0,33$ & $\mathrm{p}>0,05$ \\
\hline $\mathrm{Hb}(\mathrm{g} / \mathrm{dl})$ & $8,83 \pm 1,48$ & $8,39 \pm 1,27$ & $8,13 \pm 0,91$ & $\mathrm{p}>0,05$ \\
$\mathrm{Ht}(\%)$ & $25,91 \pm 4,72$ & $25,07 \pm 3,74$ & $24,01 \pm 2,82$ & $\mathrm{p}>0,05$ \\
Leucócitos $\left(/ \mathrm{mm}^{3}\right)$ & $10.485 \pm 3.283$ & $11.090 \pm 3.601$ & $13.241 \pm 2.469$ & $\mathrm{p}>0,05$ \\
Plaquetas $\left(/ \mathrm{mm}^{3}\right)$ & $418.937 \pm 131.885$ & $416.104 \pm 103.770$ & $445.571 \pm 65.607$ & $\mathrm{p}>0,05$ \\
\hline
\end{tabular}

Os resultados foram reportados em valores de Média \pm Desvio-padrão. Foi utilizado o teste Anova para a comparação múltipla de médias entre os 3 diferentes níveis de $\mathrm{HbF}$ e os valores de $\mathrm{He}, \mathrm{Hb}, \mathrm{Ht}$, leucócitos e plaquetas.

HbF: Hemoglobina fetal ; He: Hemácias; Hb: Hemoglobina; Ht: Hematócrito

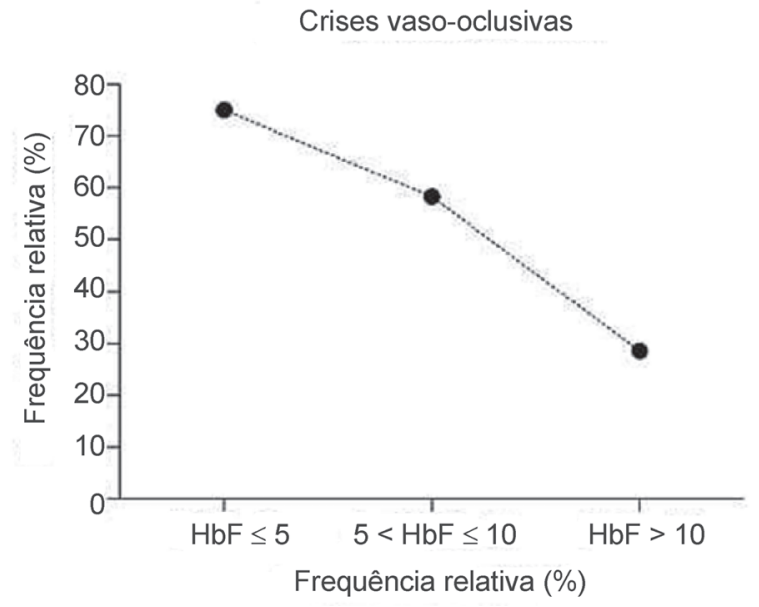

Gráfico 01- Tendência decrescente de crises vaso-oclusivas com o aumento da HbF. $p=0,0405$. A \&lt; 0

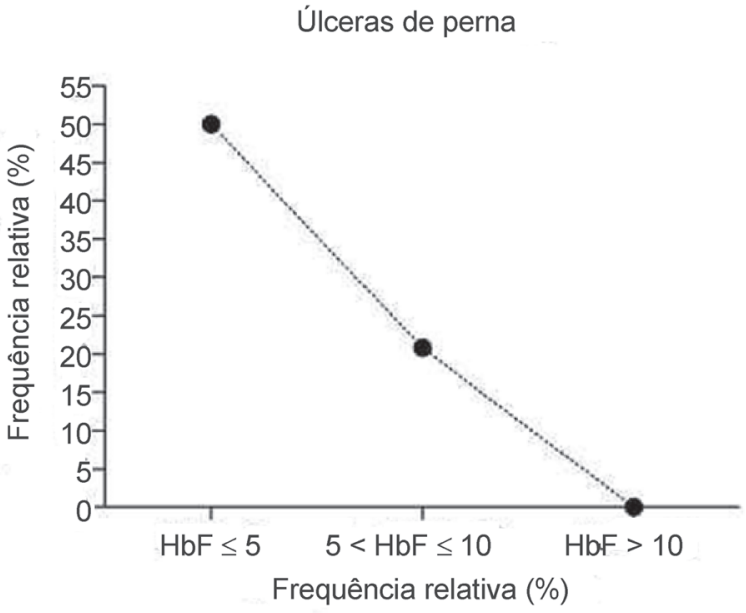

Gráfico 02- Tendência decrescente de úlceras de perna com o aumento da HbF. $p=0,0076$. A \&lt; 0 resultados também estão de acordo com os estudos de Nagel e Steinberg (2001), que também mostraram que níveis elevados de $\mathrm{HbF}$ estão associados com sintomas mais leves na anemia falciforme.

Não foi demonstrado no presente estudo a associação entre os níveis de $\mathrm{HbF}$ e os valores de $\mathrm{He}, \mathrm{Hb}, \mathrm{Ht}$, leucócitos e plaquetas $(\mathrm{p}>0,05)$. Resultado semelhante foi encontrado por Borba et al. (2003) e Adorno et al. (2008).

Os resultados demonstram que os níveis de hemoglobina fetal podem ser utilizados como fator prognóstico nos pacientes com anemia falciforme, no entanto não deve ser a única ferramenta diagnóstica para prever o quadro clínico destes pacientes.

\section{Abstract}

The fetal hemoglobin $(\mathrm{HbF})$ levels of 47 patients with sickle cell anemia living in Fortaleza, Brazil were analyzed to provide information on the influence of $\mathrm{HbF}$ on the prognosis of this disease. Measurement of hematological variables was achieved using a Sysmex KX-21N cell counter (Roche) and the levels of HbF were determined by the alkali denaturation technique. With increased levels of $\mathrm{HbF}$, there was a trend that fewer patients had painful episodes $(p=0.0405)$ and leg ulcers $(p=0.0076)$. There were no statistically significant differences between the levels of $\mathrm{HbF}$ and the concentrations of red blood cells, hemoglobin, hematocrit, leukocytes and platelets. The results show that levels of fetal hemoglobin can be used as a prognostic factor in patients with sickle cell anemia however they should not be the only diagnostic tool to predict the clinical status of these patients. Rev. Bras. Hematol. Hemoter.

Key words: Sickle cell anemia; Fetal hemoglobin; prognosis. 


\section{Referências Bibliográficas}

1. Gonçalves MS, Bomfim GC, Maciel E, Cerqueira I, Lyra I, Zanette A, et al. BetaS-haplotypes in sickle cell anemia patients from Salvador, Bahia, Northeastern Brazil. Braz J Med Biol Res. 2003;36(10):1283-8.

2. Bonini-Domingos CR. Metodologias Laboratoriais para o diagnóstico de hemoglobinopatias e talassemias. Brasil: HN, Inc. 121 p., 2006.

3. Bunn HF, Forget BG. Hemoglobin: molecular, genetic and clinical aspects. Philadelphia: W.B. Saunders Company, Inc. 690 p., 1986.

4. Weatherall DJ, Provan AB. Red cells I: inherited anaemias. Lancet. 2000;355(9210):1169-75.

5. Zago MA. Considerações gerais. In: Anvisa (Agência Nacional de Vigilância Sanitária). Manual de diagnóstico e tratamento de doenças falciformes. Brasil: Copyright. p 7-12, 2002.

6. Zago MA, Pinto ACS. Fisiopatologia das doenças falciformes: da mutação genética à insuficiência de múltiplos órgãos. Rev bras hematol hemoter. 29:207-214, 2007.

7. Steinberg MH. Modulation of fetal hemoglobin in sickle cell anemia. Hemoglobin. 2001;25(2):195-211.

8. Adorno EV, Zanette A, Lyra I, Seixas MO, Reis MG, Gonçalves MS. Clinical and molecular characteristics of sickle cell anemia in the northeast of Brazil. Genet Mol Biol. 31: 621-625, 2008.

9. Figueiredo MS. Agentes indutores da síntese de hemoglobina fetal. Rev bras hematol hemoter. 29: 313-315, 2007.

10. Steinberg MH, Barton F, Castro O, Pegelow CH, Ballas SK, Kutlar A, et al. Effect of hydroxyurea on mortality and morbidity in adult sickle cell anemia: risks and benefits up to 9 years of treatment. JAMA. 2003;289(13):1645-51.

11. Stuart MJ, Nagel RL. Sickle-cell disease. Lancet. 2004;364(9442): 1343-60.

12. Betke K, Marti HR, Schlicht I. Estimation of small percentages of foetal haemoglobin. Nature. 1959;184(Suppl 24):1877-8.

13. Buchanan GR, DeBaun MR, Quinn CT, Steinberg MH. Sickle cell disease. Hematology Am Soc Hematol Educ Program. 2004: $35-47$.

14. Nagel RL, Steinberg MH. Genetics of $\beta S$ gene: origins, genetic, epidemiology, and epistasis in sickle cell anemia. In: Steinberg MH, Forget BG, Higgs DR, Nagel R. (eds). Disorders of hemoglobingenetics, pathophisiology and clinical management. NY, USA, p. 711-755, 2001.

15. Borba R, Lima CS, Grotto HZ. Reticulocyte parameters and hemoglobin $\mathrm{F}$ production in sickle cell disease patients undergoing hydroxyurea therapy. J Clin Lab Anal. 2003;17(2):66-72.

Avaliação: Editor e dois revisores externos

Conflito de interesse: sem conflito de interesse

Recebido: 31/03/2009

Aceito após modificações: 16/04/2009 